ORIGINAL ARTICLE

\title{
KPT-8602, a second-generation inhibitor of XPO1-mediated nuclear export, is well tolerated and highly active against AML blasts and leukemia-initiating cells
}

\author{
J Etchin ${ }^{1,2}$, A Berezovskaya ${ }^{1}$, AS Conway ${ }^{1}$, IA Galinsky ${ }^{3}$, RM Stone ${ }^{3}$, E Baloglu ${ }^{4}$, W Senapedis ${ }^{4}$, Y Landesman ${ }^{4}$, M Kauffman $^{4}$, S Shacham $^{4}$, \\ JCY Wang ${ }^{5,6}$ and AT Look ${ }^{1,2}$
}

\begin{abstract}
Acute myeloid leukemia (AML) is a clonal hematologic malignant disease of developing myeloid cells that have acquired aberrant survival, uncontrolled proliferation and a block in normal hematopoietic cell differentiation. Standard chemotherapy often induces remissions in AML patients, but the disease frequently relapses due to incomplete targeting of leukemia-initiating cells (LICs), emphasizing the need for novel effective treatments. Exportin 1 (XPO1)-mediated nuclear export, which is inhibited by the drug selinexor, is an attractive new therapeutic target in AML. Selinexor has shown impressive activity in Phase I/II clinical trials for AML. Here we report the anti-leukemic efficacy and tolerability of KPT-8602, a second-generation XPO1 inhibitor. KPT-8602 demonstrates substantially reduced brain penetration compared to selinexor, with resultant attenuation of the central nervous system mediated side effects of anorexia and weight loss. Due to its improved tolerability profile, KPT-8602 can be given daily compared to the two or three times weekly regimen of selinexor, and exhibits greater anti-leukemic efficacy against both leukemic blasts and LICs in AML patient-derived xenograft models. Importantly, normal hematopoietic stem and progenitor cell (HSPC) frequency is not significantly reduced by KPT-8602, providing a therapeutic window for elimination of relapse-driving LICs while sparing normal HSPCs. These findings strongly endorse clinical testing of KPT-8602 in patients with relapsed and refractory AML.
\end{abstract}

Leukemia (2017) 31, 143-150; doi:10.1038/leu.2016.145

\section{INTRODUCTION2}

Currently available combination chemotherapy for acute myeloid leukemia (AML) induces complete remission in about $75 \%$ of younger and $45 \%$ of older patients, but the majority of patients relapse. ${ }^{1}$ This high relapse rate is likely due to the inability of the existing drugs to effectively target the self-renewing and diseasesustaining leukemia-initiating cells (LICs). ${ }^{2-5}$ Therefore, novel therapies that not only eliminate the AML blasts but also target the LICs are needed to prevent leukemic relapse.

An emerging therapeutic strategy in $\mathrm{AML}$ is inhibition of the nuclear export protein, exportin 1 (XPO1). This strategy exploits the dependence of leukemic cells on the continuous nuclear export of a subset of proteins and RNAs, although the precise cargos that are oncorequisite for AML cell survival have not yet been defined. XPO1, a karyopherin $\beta$ family member, is a major nuclearcytoplasmic exporter in eukaryotes and regulates transfer of $\sim 220$ proteins that contain leucine-rich nuclear export signals, as well as nuclear export signal-containing protein adaptors that mediate transfer of RNAs. ${ }^{6-9}$ XPO1 affinity for its cargo is mediated by binding of Ran-GTP in the nucleus, and its cargoes are released upon Ran-GTP hydrolysis by Ran-GAP ${ }^{10-14}$ in the cytoplasm. XPO1 protein cargoes include tumor suppressor proteins, apoptosis inducers and cell cycle regulators. ${ }^{15-17}$ Based on in vivo studies of selinexor, several types of hematologic malignancies and solid tumors are dependent on XPO1-mediated nuclear export for their survival. In contrast, selinexor is well tolerated by normal cells, including blood cells and gastrointestinal epithelia. ${ }^{18-26}$

In an effort to exploit this selective vulnerability of cancer cells to inhibition of nuclear export, novel small-molecule XPO1 inhibitors, or Selective Inhibitor of Nuclear Export (SINE) molecules, were designed by in silico modeling strategies. ${ }^{27}$ SINE compounds covalently bind to cysteine ${ }^{528}$ in the cargobinding groove of XPO1 to block the interaction of XPO1 with its cargo and hence inhibit nuclear export. The first-generation orally bioavailable clinical SINE compound, selinexor (KPT-330), is currently being evaluated in Phase 1 and 2 clinical trials in adult patients with AML (NCT01607892, NCT02088541, NCT02249091, NCT02403310, NCT02093403, NCT02485535, NCT02299518, NCT02416908, NCT02212561 and NCT01607892), in a Phase 1 trial for relapsed childhood ALL and AML (NCT02091245), in Phase $1 / 2$ trials in children with leukemia or MDS (NCT02212561), and in many other types of human cancer (http://clinicaltrials.gov). The results of these trials are encouraging, as they have demonstrated that selinexor alone or in combination with conventional therapies is active in inducing remission in patients with relapsed or refractory $\mathrm{AML}^{22,28-31}$

Studies by our group and others demonstrated the antileukemic activity of selinexor in xenograft preclinical models of human hematologic malignancies, including $\mathrm{AML}$, chronic lymphocytic leukemia, T-cell acute lymphoblastic leukemia, chronic lymphocytic leukemia, and multiple myeloma. ${ }^{18,19,22,23,25,32-38}$

${ }^{1}$ Department of Pediatric Oncology, Dana-Farber Cancer Institute, Boston, MA, USA; ${ }^{2}$ Division of Hematology/Oncology, Children's Hospital, Boston, MA, USA; ${ }^{3}$ Department of Medical Oncology, Dana-Farber Cancer Institute, and Harvard Medical School, Boston, MA, USA; ${ }^{4}$ Karyopharm Therapeutics, Newton, MA, USA; ${ }^{5}$ Princess Margaret Cancer Centre, University Health Network, Toronto, ON, Canada and ${ }^{6}$ Department of Medicine, University of Toronto, Toronto, ON, Canada. Correspondence: Professor Dr AT Look, Department of Pediatric Oncology, Dana-Farber Cancer Institute, Mayer Building, Room 630, 450 Brookline Avenue, Boston, 02215 MA, USA. E-mail: thomas_look@dfci.harvard.edu

Received 8 January 2016; revised 24 March 2016; accepted 25 April 2016; accepted article preview online 23 May 2016 ; advance online publication, 24 June 2016 
144

In our most recent published study, we tested the activity of selinexor in primary patient-derived xenografts, or PDX models, established from AML patient cell samples. ${ }^{39-41}$ PDX models are the gold standard assay for assessing the self-renewing LIC population, and the properties of LICs that read out in these assays have been shown to be clinically relevant. ${ }^{42,43}$ In PDX models established from high-risk AML patients, selinexor very potently inhibits human AML blasts and eliminates the self-renewing LICs. $^{40}$

Recently, a second-generation SINE compound, KPT-8602, was discovered by drug metabolism and pharmacokinetics-directed drug design (Supplementary Figure 1). KPT-8602 is orally bioavailable and has similar pharmacokinetic properties to selinexor, but has markedly reduced (approximately 30 -fold less) penetration across the blood-brain barrier, as demonstrated in Table 1 of the accompanying manuscript by Hing et al. In clinical trials, the dose-limiting side effects of selinexor are central nervous system (CNS)-mediated anorexia with weight loss and malaise, as well as thrombocytopenia, which limit the frequency of dosing to two or three times per week. Toxicology studies in rats and monkeys indicate that KPT-8602 has a substantially better tolerability profile, probably due to its inability to penetrate into the CNS, with reduced anorexia, malaise and weight loss compared to selinexor. This evidence prompted us to test the anti-leukemic activity of KPT-8602 against LICs in AML, and to compare the efficacy and tolerability of KPT-8602 and selinexor in PDX models of high-risk AML. Our results demonstrate the superior anti-leukemic efficacy of KPT-8602 compared to selinexor, with minimal toxicity to normal hematopoietic stem and progenitor cells. These findings indicate that KPT-8602 should be tested for efficacy and tolerability in clinical trials of patients with relapsed and refractory AML.

\section{MATERIALS AND METHODS}

Patient-derived xenograft models of AML

Primary patient cells were collected from bone marrow aspirates of AML patients according to approved protocols. We used low-passage human AML cells that have been expanded from patient AML cells by injection into NOD-SCID-IL2Rcy null (NSG) mice (but never cultured in tissue culture) and cryopreserved in multiple vials for experiments. To establish murine models of patient AML, or patient-derived xenograft (PDX) model, primary leukemic cells from AML patients were injected via the tail vein into NSG mice (The Jackson Laboratory, Bar Harbor, ME, USA). Mice received the same number of AML cells, ranging from $8 \times 10^{4}$ to $20 \times 10^{4} \mathrm{AML}$ cells per mouse, for establishing each PDX model. Injected mice were monitored for human CD45-positive ( $\mathrm{hCD} 45+$ ) leukemia cells in peripheral blood by flow cytometry every 2 weeks and treatment was initiated when more than $5 \%$ of the cells were hCD45+ AML cells, as described previously. ${ }^{40}$ To determine the level of leukemia infiltration and to obtain absolute counts (hCD45+ cells per femur and tibia) prior to therapy, four mice were killed and the bone marrow cells were extracted from femurs, tibia or spine by crushing the bone or spleen in FBS-supplemented RPMI medium. To determine the drug efficacies, mice were dosed by oral gavage with vehicle (methylcellulose/tween 80 ), selinexor $(20 \mathrm{mg} / \mathrm{kg}$ twice a week for PDX AMLCN and three times a week for AML-CK and AML/MDS) or KPT-8602 (15 mg/kg daily) for 4 weeks. To determine the drug response of mice in all experimental groups, we measured the percent hCD45+ AML cell infiltration among the bone marrow leukocytes and absolute counts of hCD45+ cells per femur and tibia in the PDX models of each experimental group.

\section{Secondary transplantation assays}

After 4 weeks of treatment, we killed the PDX mice and isolated the femur and tibia bone marrow cells in 4-7 mice of each experimental group. The bone marrow hCD45+ AML blasts at doses from $10^{6}$ to $10^{2}$ hCD45+ cells were transplanted into new recipient mice. After $18-22$ weeks, cohorts of mice transplanted with dilutions of hCD45+ cells were screened for hCD45+ infiltration into the bone marrow of mouse femurs (for details please see reference 33 in Etchin et $a l^{40}{ }^{0}$. The fractions of leukemic mice per total number of mice in each dilution cohort were used to calculate the LIC frequency by Extreme Limiting Dilution software (http://bioinf.wehi.edu.au/ software/elda/). Please see Schmidt et al. ${ }^{33}$ in the main text for details.

\section{Engraftment of normal hCD34+ cells into NSG mice}

Human CD34+ (hCD34+) cells were isolated from the cord blood of consenting healthy mothers according to approved procedures. $10^{5} \mathrm{hCD} 34+$ cells per mouse were intravenously injected into sublethally (200 cGy) irradiated NSG mice and peripheral blood was analyzed for the appearance of hCD45+ cells by flow cytometric analysis every 2-4 weeks. Human grafts were treated with either vehicle, selinexor $(20 \mathrm{mg} / \mathrm{kg}$ twice a week for PDX AML-CN and three times a week for AML-CK and AML/MDS) or KPT-8602 (15 mg/kg daily) for 4 weeks. After 4 weeks of treatment, mice were killed and bone marrow was isolated from the femurs and tibias of 5-7 animals from each experimental group. The drug response of mice in each experimental group was measured by the percent infiltration of hCD45+ cells among the bone marrow leukocytes and total hCD45+ cell per femur and tibia. To determine the toxicity of drugs against hematopoietic stem cells (HSCs), serial dilutions of hCD34+ cells isolated from the bone marrow of femurs and tibias of mice from each experimental group after 4 weeks of treatment were transplanted into new recipient animals. After $18-22$ weeks, cohorts of mice transplanted with dilutions of hCD45+ cells were analyzed for the presence of hCD45+ in the mouse bone marrow, following the same procedures as described for secondary transplantation assays for the analysis of LIC frequencies. The fractions of mice that demonstrated hCD34+ engraftment per total number of animals in each dilution cohort were used to determine the HSC frequency by Extreme Limiting Dilution software. The following antibodies were used for flow cytometric analysis: hCD45, hCD33 (Biolegend, San Diego, CA, USA), hCD34 and hCD38 (BD Biosciences, San Jose, CA, USA).

\section{Statistical analysis}

Percent and total hCD45+ cells in the bone marrow are presented as means \pm s.e.m. Differences between experimental groups were determined with an unpaired Student's $t$-test. LIC and HSC frequencies were estimated from results of the secondary transplantation assays using Extreme Limiting Dilution software (http://bioinf.wehi.edu.au/software/elda/). ${ }^{44}$

\section{RESULTS}

KPT-8602 demonstrates anti-leukemic activity in vitro

We initiated our study of a second-generation XPO1 inhibitor, KPT-8602, by examining the effects of KPT-8602 on the viability of human AML cell lines in vitro. Dose - response measurements of $10 \mathrm{AML}$ lines incubated with KPT-8602 yielded $\mathrm{IC}_{50}$ values of 20-211 nM after 3 days of exposure, as determined by Cell Titer Glo viability assay (Table 1). These data demonstrate that KPT-8602 is a potent inhibitor of AML cells in cell-based viability assays. For each cell line, the half-maximal inhibitory value $\left(\mathrm{IC}_{50}\right)$ is consistently $30-50 \%$ lower for KPT-8602 than for selinexor on a nanomolar basis, indicating that KPT-8602 is more active than the first-generation XPO1 inhibitor, selinexor (Table 1).

\begin{tabular}{|c|c|c|c|}
\hline AML cell lines & $\begin{array}{c}K P T-8602-I C_{50}(n м) \\
\text { at } 72 h\end{array}$ & $\begin{array}{c}\text { Selinexor- }-I C_{50}(n м) \\
\text { at } 72 h\end{array}$ & Ratio \\
\hline OCI-AML2 & 19 & 40 & 0.48 \\
\hline MV411 & 30 & 50 & 0.61 \\
\hline MOLM13 & 32 & 47 & 0.68 \\
\hline KG1 & 66 & 151 & 0.44 \\
\hline SKM1 & 84 & 120 & 0.70 \\
\hline Kasumi-1 & 113 & 172 & 0.65 \\
\hline OCI-AML3 & 127 & 154 & 0.82 \\
\hline Mono-Macl & 167 & 179 & 0.93 \\
\hline U937 & 205 & 327 & 0.62 \\
\hline NB4 & 211 & 304 & 0.69 \\
\hline
\end{tabular}

Abbreviation: $\mathrm{IC}_{50}, 50 \%$ inhibitory concentration 
KPT-8602 is highly active against AML blast cells in PDX models To determine the activity of KPT-8602 in human AML, we employed PDX models in which patient leukemic blast cells were transplanted into immunodeficient NOD-SCID-IL2Rcy null (NSG) mice. The three primary AMLs selected for this study were from high-risk $A M L$ groups: complex karyotype $A M L(A M L-C K)$, normal cytogenetics AML (AML-CN) in which the blast cells harbored a FLT3 internal tandem duplication (FLT3-ITD) and MDS-derived AML (MDS/AML). ${ }^{1,45}$

For each PDX model of human patient $A M L$, leukemic blast cells were injected intravenously into NSG mice and monitored for disease progression, as demonstrated by the appearance of human $\mathrm{CD} 45+(\mathrm{hCD} 45+)$ cells in peripheral blood. Analysis of the extent of hCD45+ cell infiltration in the femur and tibia bones extracted from four engrafted animals was used to confirm that they harbored a heavy disease burden prior to the start of therapy. Once disease burden was established, the mice were split into vehicle control, selinexor and KPT-8602 experimental groups, and one of the drugs or vehicle was administered by oral gavage (Figure 1). At the end of the treatment period, response of the bulk AML cells was measured by percent hCD45+ cell infiltration and total counts of hCD45+ cells per femur plus tibia in the mice of each experimental group (Figure 1).

KPT-8602 is much better tolerated by mice than selinexor. Specifically, selinexor given at $20 \mathrm{mg} / \mathrm{kg}$ three times a week for 4 weeks causes dose-limiting anorexia and weight loss. In contrast, KPT-8602 given at $15 \mathrm{mg} / \mathrm{kg}$ daily for 4 weeks did not cause any weight loss in mice and bacon flavoring was not needed to encourage the mice to eat. Analysis of the anti-leukemic activity of the drugs demonstrated that although both selinexor and KPT-8602 were cytotoxic to leukemic blasts in all PDX models tested, KPT-8602 exhibited substantially more profound antileukemic activity in AML-CN and AML-CK PDX mice (Figure 2). Specifically, in the AML-CK PDX model, the control mice show hCD45+ cell infiltration levels of $91 \pm 0.9 \%$ of bone marrow leukocytes, with total hCD45+ cell counts of $5.3 \times 10^{6} \pm 0.2 \times 10^{6}$ per femur and tibia (Figure 2a). Selinexor caused a reduction in hCD $45+$ cell infiltration to $49 \pm 8.8 \%$ and a 3.5 -fold decrease in total hCD45+ cell counts to $1.5 \times 10^{6} \pm 0.4 \times 10^{6}$. In KPT-8602-treated mice, there was a more remarkable decrease in AML blasts, with hCD45+ cells accounting for only $7.6 \pm 1.0 \%$ of bone marrow leukocytes and $0.2 \times 10^{6} \pm 0.05 \times 10^{6}$ hCD $45+$ cells per femur and tibia ( 27-fold reduction in AML cell numbers; Figure $2 \mathrm{a}$ ). In the AML-CN PDX model, both drugs promoted a leukemic blast reduction; however, KPT-8602 demonstrated higher anti-leukemic activity than selinexor, with no leukemic cells detected in the bone marrow of two of the eight AML-CN PDX-treated mice (Figure 2b). Taken together, these data demonstrate that KPT-8602 exhibits superior anti-leukemic activity and better tolerability in the AML PDX models tested, with nearly complete elimination of human AML cells in the AML-CN model.

\section{KPT-8602 effectively targets the LICs of PDX models of primary} AML

We next examined the effects of KPT-8602 on AML LIC in the three PDX models. To determine the frequency of LICs in the vehicle control, selinexor or KPT-8602, we used a limiting dilution transplantation assay (Experimental Scheme, Figure 3a). Briefly, the bone marrow cells from the femurs and tibias of PDX mice after the treatment period were analyzed for hCD45+, and the cells were retransplanted at serial dilutions by i.v. injection into new NSG mice that were then left untreated (Figure 3a). After 18-22 weeks, or earlier if the mice became ill, the recipient animals were analyzed for measurable hCD45+ AML cells in the mouse bone marrow. The fraction of mice with any detectable hCD45+ cells in each transplantation group determined the LIC frequency for the vehicle, selinexor and KPT-8602 treatment groups for each of the three AML PDX models. ${ }^{44}$ The results of the limiting dilution transplantation assays clearly show that although both selinexor and KPT-8602 effectively target AML LICs, KPT-8602 is more potent in eliminating LICs, as evidenced by a more profound reduction of LIC frequency in the AML-CK and AML-CN PDX models (507-fold vs 0.9-fold reduction in AML-CK, $P<0.0001$; and 437-fold vs 111 -fold reduction in AML-CN by KPT-8602 vs selinexor, $P=0.79$ ) (Figure $3 b$ ). In the MDS/AML PDX model, the anti-leukemic efficacy against LICs was comparable for both drugs (Figure 3b). Thus, KPT-8602 effectively eliminates LICs from highrisk AML patients.

Selinexor and KPT-8602 greatly extend the survival of PDX mice We next assessed if prolonged treatment with selinexor or KPT-8602 extends the survival of PDX mice. Briefly, mice engrafted with either AML-CK or MDS/AML were treated with vehicle, selinexor or KPT-8602 for 4 months or until they became ill. After the treatment phase, the mice were monitored for ruffled fur and reduced activity and eventually were assessed for hCD45+ infiltration in the bone marrow or spleen. AML-CK mice treated

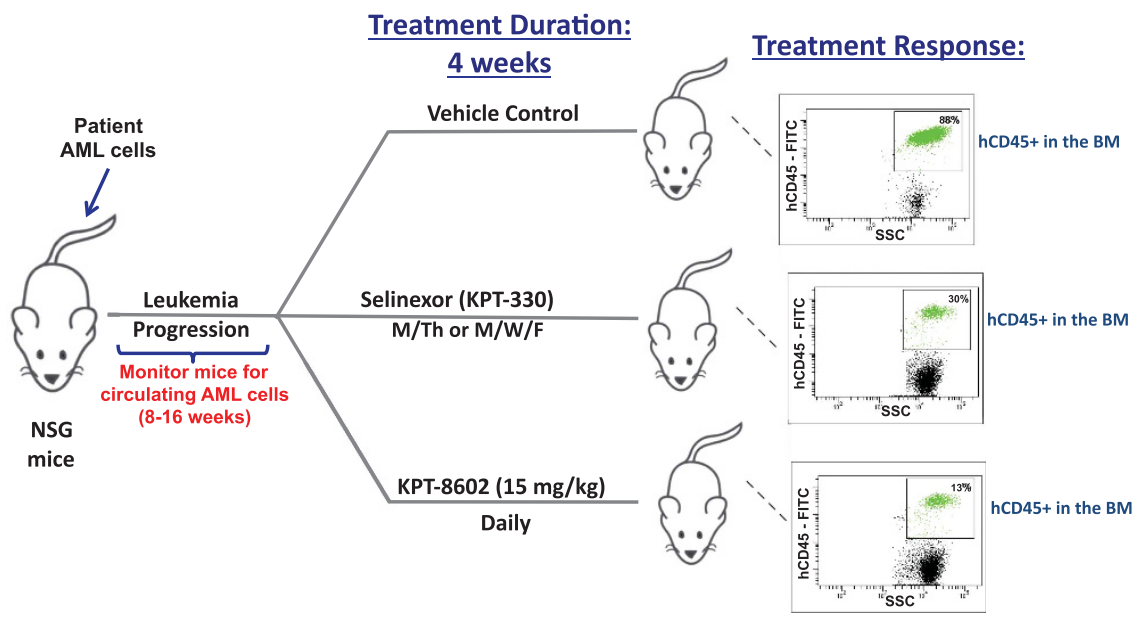

Figure 1. Experimental outline for the analysis of the anti-leukemic activity of KPT-8602 against primary human AML cells. Patient-derived xenograft mouse models of human primary AML were used in therapeutic experiments to assess the efficacy of KPT-8602 against AML cells isolated from patients with AML-CN, AML-CK and AML/MDS. BM, bone marrow. 
a

AML-CK
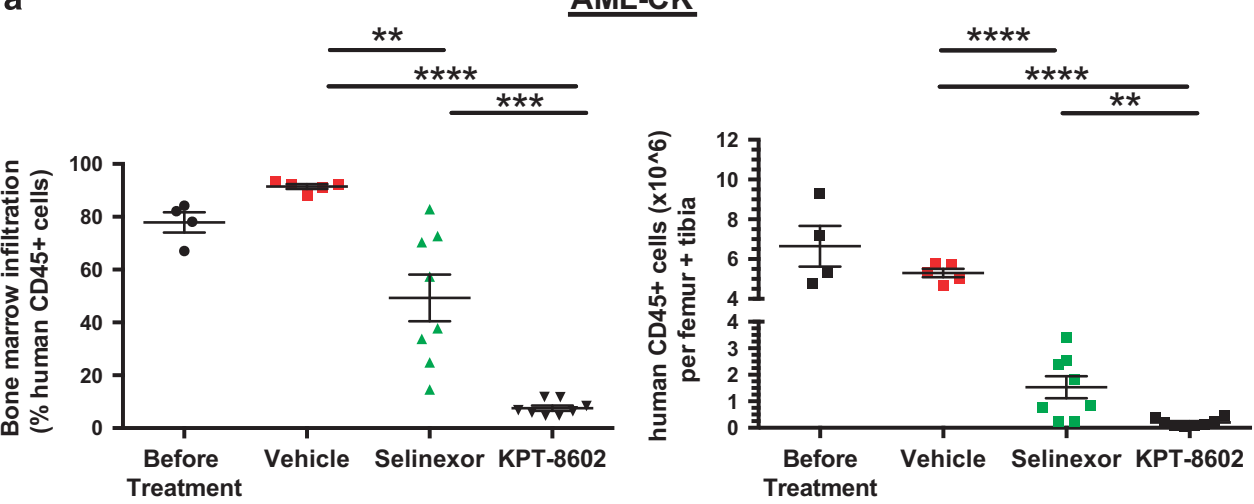

b

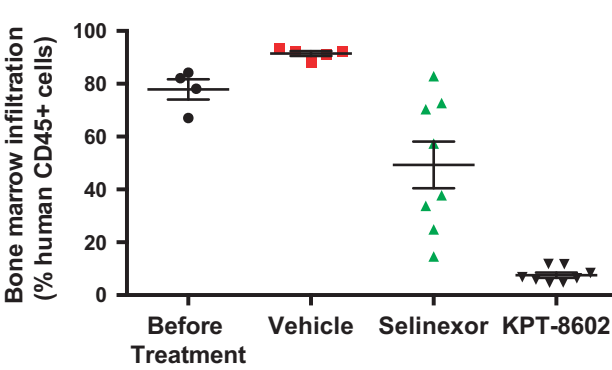

AML-CN
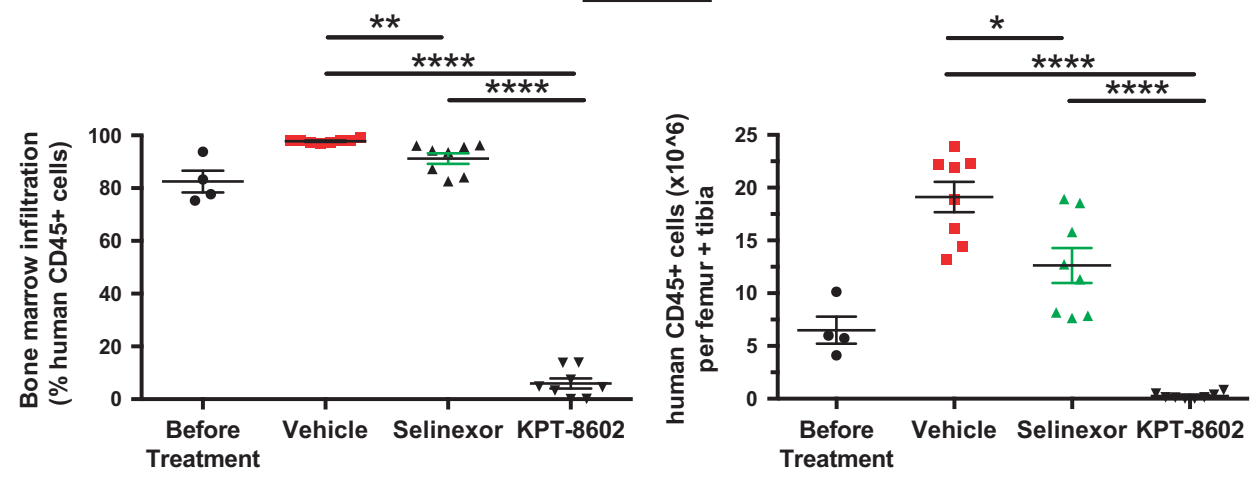

C

AML/MDS
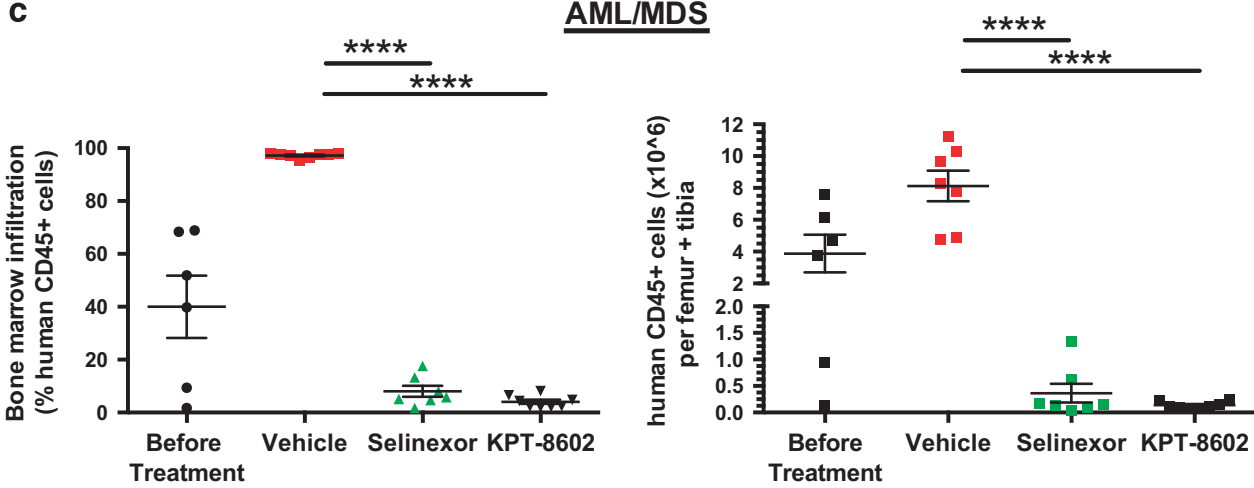

Figure 2. KPT-8602 is highly active against primary AML-CN, AML-CK and AML/MDS cells transplanted into NSG mice. AML cells isolated from patients with normal karyotype AML (AML-CN), complex karyotype AML (AML-CK) and AML/MDS were engrafted into NSG mice for therapeutic experiments. On the left panel of the figure, percent infiltration of human patient AML (human CD45+) cells in the bone marrow of NSG mice before and after treatment with vehicle, selinexor or KPT-8602. On the right panel, counts of human CD45+ cells per femur and tibia in mice transplanted with AML cells before treatment and following treatment with vehicle, selinexor or KPT-8602. Each symbol denotes a single animal ( $n=4-8$ per group). Error bars represent mean \pm s.e.m.; ${ }^{*} P<0.05,{ }^{* *} P<0.01,{ }^{* * *} P<0.001,{ }^{* * *} P<0.0001$ by unpaired $t$-test for comparisons between the indicated groups.

with vehicle succumbed to disease within 1 month of treatment (Figure 4a). In this PDX model, selinexor extended survival to $50-60$ days from the treatment start. Strikingly, mice treated with KPT-8602 were still alive at $\sim 140$ days after the start of treatment, when the experiment was terminated (Figure 4a). Upon postmortem analysis, residual hCD45+ cells were detected in the mouse spleens but not in the bone marrow. In the MDS/AML group, vehicle-treated mice became moribund $\sim 40-90$ days from the start treatment, and mice treated with either selinexor or KPT-8602 for 17 weeks were alive 29 weeks from the start of treatment, when the experiment was terminated. Upon postmortem analysis, no AML cells were detected in the spleen or bone marrow of seven of seven mice treated with selinexor and four of seven mice treated with KPT-8602 ( $P=0.19$ indicating similar survival benefit between the selinexor and KPT-8602 treatment groups).

KPT-8602 is minimally toxic to normal hematopoietic stem and progenitor cells

To define the hematopoietic toxicity of KPT-8602, we engrafted NSG mice with human CD34+ (hCD34+) cord blood cells. Successful engraftment of human blood cells was documented 

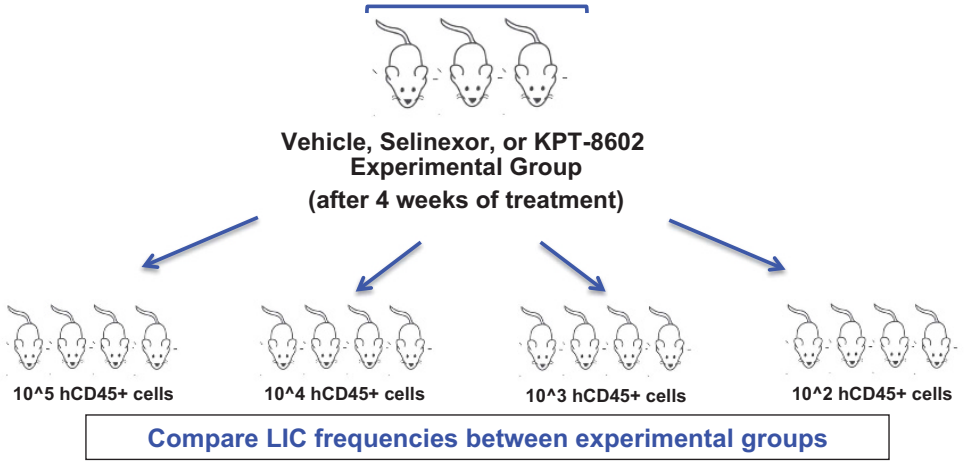

b

\begin{tabular}{|l|c|c|c|c|c|c|}
\hline & \multicolumn{2}{|c|}{ PDX model of AML-CN } & \multicolumn{2}{c|}{ PDX model of AML-CK } & \multicolumn{2}{c|}{ PDX model of MDS/AML } \\
\hline & $\begin{array}{c}\text { LIC } \\
\text { Frequency }\end{array}$ & $\begin{array}{c}\text { Fold Reduction } \\
\text { in LIC } \\
\text { Frequency* }\end{array}$ & $\begin{array}{c}\text { LIC } \\
\text { Frequency }\end{array}$ & $\begin{array}{c}\text { Fold Reduction } \\
\text { in LIC } \\
\text { Frequency* }\end{array}$ & $\begin{array}{c}\text { LIC } \\
\text { Frequency }\end{array}$ & $\begin{array}{c}\text { Fold Reduction } \\
\text { in LIC } \\
\text { Frequency* }\end{array}$ \\
\hline Vehicle & $1 / 1155$ & 1 & $1 / 311$ & 1 & $1 / 4092$ & 1 \\
\hline Selinexor & $1 / 128923$ & $\downarrow 111$-fold & $1 / 280$ & $\downarrow 0.9$-fold & $<1 / 771218$ & $\downarrow>150$-fold \\
\hline KPT-8602 & $1 / 504215$ & $\downarrow 437$-fold & $1 / 157733$ & $\downarrow 507$-fold & $<1 / 681463$ & $\downarrow>150$-fold \\
\hline
\end{tabular}

Figure 3. KPT-8602 efficiently targets the LICs of three primary AML samples engrafted into NSG mice. (a) Scheme for the limiting dilution repopulation assay. After 4 weeks of treatment, bone marrow AML cells isolated from mice treated with either vehicle, selinexor or KPT-8602 were re-transplanted at serial dilutions into new recipient animals. The number of leukemic mice per total number of animals injected with AML cells in secondary recipients was used to determine the LIC frequency in vehicle- and drug-treated AML cell populations. (b) The table shows the LIC frequencies in AML-CN, AML-CK and AML/MDS xenografts after treatment with vehicle, selinexor or KPT-8602. The fold reduction in LIC frequency in response to drug treatment compared to vehicle controls shows the extent of LIC elimination in response to drug therapy.

a

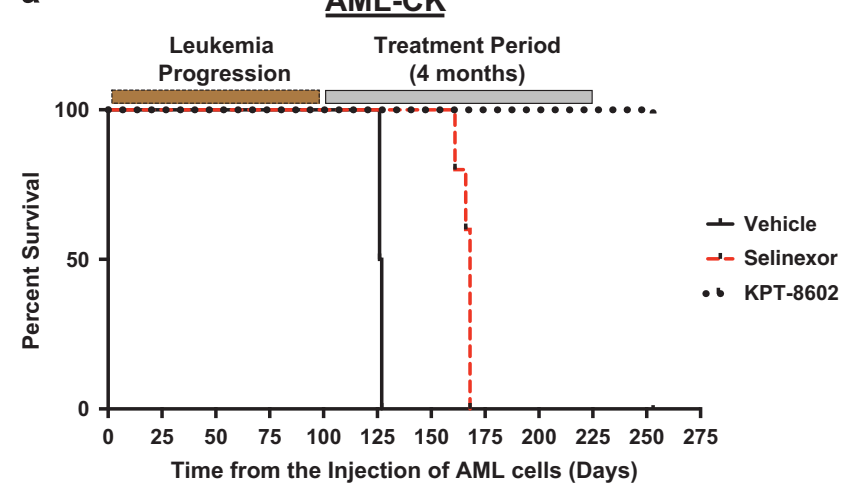

b

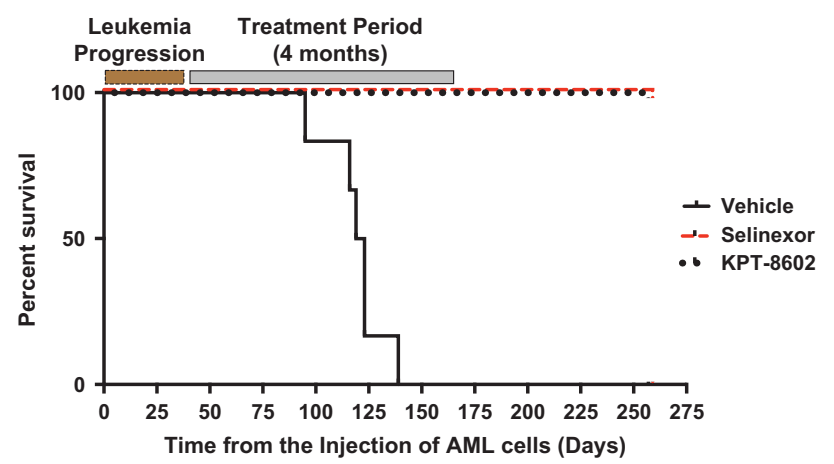

Figure 4. KPT-8602 greatly extends the survival of AML patientderived xenografts (PDXs). Kaplan - Meier curves of PDX models of MDS/AML (a) and AML-CK (b) following treatment with vehicle, selinexor or KPT-8602. The engrafted mice were allowed to become leukemic with circulating AML cells and then treated for 4 months. MDS/AML and AML-CK PDX mice were followed for additional 1-3 months to see if regrowth of AML could be prevented or delayed by longer treatments. (a) In the AML-CK PDX model, mice treated with KPT-8602 were alive at day 253. In these mice, no leukemia cells were detected in the bone marrow. (b) In the PDX model of MDS/AML, both selinexor- and KPT-8602-treated mice were alive at day 252. No leukemia cells were detected in the bone marrows of seven out of seven mice treated with selinexor and four out of seven mice treated with KPT-8602 $(P=0.19$; Fisher's exact test).

by the presence of $>30 \%$ hCD45+ cells in the mouse peripheral blood. Engrafted mice were dosed orally with vehicle or KPT-8602 $(15 \mathrm{mg} / \mathrm{kg})$ daily for 4 weeks. After treatment, the bone marrows of KPT-8602-treated mice had a six-fold reduction in hCD45+ cell numbers per femur and tibia compared to vehicle-treated mice (Figure 5a). The impact on normal human hematopoietic stem and progenitor cell fractions by CD34/CD38 flow analysis and retransplantation assays (performed by the same methodology as described for PDX models of AML) demonstrates that, in contrast to its profound effects against AML LIC, KPT-8602 does not preferentially kill normal hematopoietic stem and progenitor cells (Figures $5 b$ and $c$ ). In future studies of this drug, it will be important to further fractionate hematopoietic stem and progenitor cells using newly developed markers that distinguish functional subsets of these cells. ${ }^{46}$ In addition, murine neutrophil 

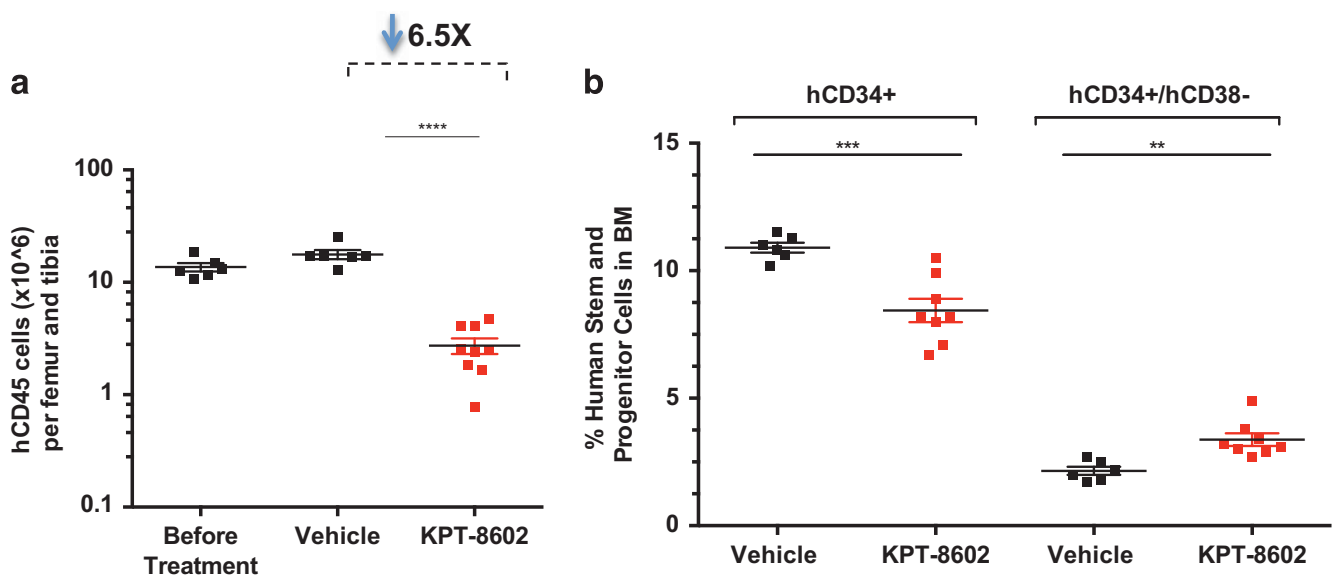

c

\begin{tabular}{|c|c|c|}
\hline $\begin{array}{c}\text { Normal Human } \\
\text { CD34+ Grafts }\end{array}$ & $\begin{array}{c}\frac{\text { Secondary }}{\text { Transplant }} \\
\text { Normal HSC } \\
\text { frequency }\end{array}$ & $\begin{array}{c}\text { Ratio of HSC Frequency } \\
\text { in Drug vs. Vehicle Groups }\end{array}$ \\
\hline Vehicle & $1 / 7389$ & 1 \\
\hline Selinexor & $1 / 20496$ & 2.77 \\
\hline KPT-8602 & $1 / 6264$ & 0.85 \\
\hline
\end{tabular}

Figure 5. KPT-8602 spares normal human hematopoietic stem and progenitor cells (HSPCs) engrafted into NSG mice. (a) Human CD45+ (hCD45+) cells per femur and tibia in normal human CD34+ grafts before and after treatment with vehicle or KPT-8602 for 4 weeks. (b) Percentage of hematopoietic stem and progenitor cells, hCD34+ and hCD34+/CD38 - cells, in the BM of established grafts treated with vehicle or KPT-8602. (c) Normal HSC frequencies in grafts treated with vehicle, selinexor or KPT-8602 were obtained from analysis after transplantation into untreated secondary recipients. Fold reduction in HSC frequencies of selinexor or KPT-8602-treated grafts compared to vehicle-treated grafts. $n=6-9$ mice per group. Error bars represent mean \pm s.e.m.; ${ }^{* * *} P<0.0001$ by Student's $t$-test for comparison between the indicated groups. ${ }^{* *} P<0.001,{ }^{* * *} P<0.0001$.

and platelet counts and hemoglobin levels were within the normal range, as demonstrated in the PDX model of AML/MDS (Supplementary Figure 2).

\section{DISCUSSION}

Our study demonstrates the anti-leukemia activity and tolerability of KPT-8602, a second-generation drug of the SINE class of XPO1mediated nuclear export inhibitors, in PDX models of high-risk human AML. Compared to selinexor, the first-generation and current clinical lead SINE drug, KPT-8602, exhibits superior efficacy against both bulk leukemic blasts and disease-sustaining LICs. KPT-8602 is an oral drug suitable for daily administration, and thus can easily be incorporated into combination regimens with current cytotoxic agents, which do not effectively target LICS, or with newer agents. The improved survival of PDX mice after prolonged treatment with KPT-8602 demonstrates the curative potential of this drug with extended administration. Importantly, KPT-8602 had minimal effects on normal hematopoietic stem and progenitor cells, suggesting that, like selinexor, it will not induce significant hematopoietic toxicity in patients. The preferential activity of KPT-8602 against AML cells, including LICs, compared to normal hematopoietic stem and progenitor cells implies that the former are more dependent for survival on XPO1mediated nuclear export of one or more macromolecular cargos. XPO1-mediated nuclear export regulates a subset of protein and RNA molecules that function in apoptotic signaling and cell cycle regulation. The mechanisms that mediate apoptotic signaling in neoplastic cells but not in normal cells due to inhibition of XPO1 activity by KPT-8602 remain to be elucidated.

The improved tolerability of KPT-8602 is likely due to its exclusion from the CNS by the blood-brain barrier, and is consistent with a central origin of the anorexia and malaise induced by selinexor. Because the blood-brain penetration of KPT-8602 relative to plasma concentrations was even less in monkeys than in mice, we are optimistic that patients treated with this drug will experience little to no nausea, anorexia and malaise, adverse effects that have been troublesome to a subset of patients receiving selinexor. Unlike ALL, CNS infiltration is not a major problem for AML patients, and CNS prophylaxis is not routinely given to AML patients undergoing treatment. Thus we do not think that the lack of CNS penetration will present a problem in terms of the anti-leukemic efficacy for AML patients treated with KPT-8602.

An IND for KPT-8602 was recently approved by the FDA (IND Number 126583) and KPT-8602 entered a Phase 1/2 Open-Label Study of the Safety, Tolerability and Efficacy of the SINE Compound KPT-8602 in Patients with Relapsed/Refractory Multiple Myeloma in January 2016. Our preclinical results indicate that KPT-8602 should be tested in clinical trials to determine its toxicity and efficacy in patients with advanced relapsed or refractory $A M L$, with the hope that it can eventually be integrated into upfront combination chemotherapy regimens. Resistance will develop to any single agent used to treat AML, but the completely novel mechanism of action of KPT-8602 in comparison with currently approved drugs suggests 
that it could be extremely active in combination, by helping to kill residual $A M L$ LICs that can drive relapse.

\section{CONFLICT OF INTEREST}

EB, WS, YL, SS and MK are employees of Karyopharm Therapeutics Incorporated and receive compensation and hold equity in the company.

\section{ACKNOWLEDGEMENTS}

We would like to thank Paul Kirschmeier and Prafulla Gokhale of the Lurie Family Imaging Center for assistance with the in vivo studies. We would like to thank the flow cytometry team led by John Daley and Suzan Lazo for their help with cell analysis. This research was supported by William Lawrence and Blanche Hughes Foundation (ATL), Leukemia and Lymphoma Society's Translational research grant (ATL), Alex's Lemonade Young Research Investigator grant (JE), Luck2Tuck Foundation (JE), Claudia Adams Barr Innovative Basic Science Research Grant (JE) and Wong Family Award in Translational Research (JE).

\section{AUTHOR CONTRIBUTIONS}

JE designed and performed the experiments, analyzed the data and wrote the manuscript. $A B$ performed in vivo mouse studies and analyzed the data. ASC performed in vivo mouse experiments. SS designed the KPT-SINE compounds. EB designed second-generation SINE, KPT-8602 compound. EB, WS, YL, SS and MK provided toxicology results and plasma vs brain measurements in mice and monkeys, and participated in the design of in vivo mouse studies and data analysis. IAG and RMS provided AML patient samples for this study. JCYW provided guidance on limit dilution transplantation studies n PDX models, analyzed the results and edited the manuscript. ATL guided the research, analyzed the results and wrote the paper.

\section{REFERENCES}

1 Estey EH. Acute myeloid leukemia: 2014 update on risk-stratification and management. Am J Hematol 2014; 89: 1063-1081.

2 Bonnet D, Dick JE. Human acute myeloid leukemia is organized as a hierarchy that originates from a primitive hematopoietic cell. Nat Med 1997; 3: 730-737.

3 Hope KJ, Jin L, Dick JE. Acute myeloid leukemia originates from a hierarchy of leukemic stem cell classes that differ in self-renewal capacity. Nat Immunol 2004; 5: 738-743.

4 Lapidot T, Sirard C, Vormoor J, Murdoch B, Hoang T, Caceres-Cortes J et al. A cell initiating human acute myeloid leukaemia after transplantation into SCID mice. Nature 1994; 367: 645-648.

5 Gilliland DG, Jordan CT, Felix CA. The molecular basis of leukemia. Hematology Am Soc Hematol Educ Program 2004; 2004: 80-97.

6 Fornerod M, Ohno M, Yoshida M, Mattaj IW. CRM1 is an export receptor for leucine-rich nuclear export signals. Cell 1997; 90: 1051-1060.

7 Fukuda M, Asano S, Nakamura T, Adachi M, Yoshida M. CRM1 is responsible for intracellular transport meditted by the nuclear export signal. Nature 1997; 390: 308-311.

8 Ossareh-Nazari B, Bachelerie F, Dargemont C. Evidence for a role of CRM1 in signal-mediated nuclear protein export. Science 1997; 278: 141-144.

9 Stade K, Ford CS, Guthrie C, Weis K. Exportin 1 (Crm1p) is an essential nuclear export factor. Cell 1997; 90: 1041-1050.

10 Dong X, Biswas A, Chook YM. Structural basis of assembly and disassembly of the CRM1 nuclear export complex. Nat Struct Mol Biol 2009; 16: 558-560.

11 Dong X, Biswas A, Suel KE, Jackson LK, Martinez R, Gu H et al. Structural basis for leucine-rich nuclear export signal recognition by CRM1. Nature 2009; 458: 1136-1141.

12 Guttler T, Gorlich D. Ran-dependent nuclear export mediators: a structural perspective. EMBO J 2011; 30: 3457-3474.

13 Hutten S, Kehlenbach RH. CRM1-mediated nuclear export: to the pore and beyond. Trends Cell Biol 2007; 17: 193-201.

14 Koyama M, Matsuura Y. An allosteric mechanism to displace nuclear export cargo from CRM1 and RanGTP by RanBP1. EMBO J 2010; 29: 2002-2013.

15 Turner JG, Dawson J, Sullivan DM. Nuclear export of proteins and drug resistance in cancer. Biochem Pharmacol 2012; 83: 1021-1032.

16 Xu D, Farmer A, Collett G, Grishin NV, Chook YM. Sequence and structural analyses of nuclear export signals in the NESdb database. Mol Biol Cell 2012; 23: 3677-3693.
17 Xu D, Grishin NV, Chook YM. NESdb: a database of NES-containing CRM1 cargos. Mol Biol Cell 2012; 23: 3673-3676.

18 Etchin J, Sanda T, Mansour MR, Kentsis A, Montero J, Le BT et al. KPT-330 inhibitor of CRM1 (XPO1)-mediated nuclear export has selective anti-leukaemic activity in preclinical models of T-cell acute lymphoblastic leukaemia and acute myeloid leukaemia. Br J Haematol 2013; 161: 117-127.

19 Etchin J, Sun Q, Kentsis A, Farmer A, Zhang ZC, Sanda T et al. Antileukemic activity of nuclear export inhibitors that spare normal hematopoietic cells. Leukemia 2013; 27: 66-74.

20 Huang WY, Yue L, Qiu WS, Wang LW, Zhou XH, Sun YJ. Prognostic value of CRM1 in pancreas cancer. Clin Invest Med 2009; 32: E315.

21 Inoue $\mathrm{H}$, Kauffman $\mathrm{M}$, Shacham S, Landesman $\mathrm{Y}$, Yang J, Evans CP et al. CRM1 blockade by selective inhibitors of nuclear export attenuates kidney cancer growth. J Urol 2013; 189: 2317-2326.

22 Kojima K, Kornblau SM, Ruvolo V, Dilip A, Duvvuri S, Davis RE et al. Prognostic impact and targeting of CRM1 in acute myeloid leukemia. Blood 2013; 121: 4166-4174.

23 Lapalombella R, Sun Q, Williams K, Tangeman L, Jha S, Zhong Y et al. Selective inhibitors of nuclear export show that CRM1/XPO1 is a target in chronic lymphocytic leukemia. Blood 2012; 120: 4621-4634.

24 Shen A, Wang Y, Zhao Y, Zou L, Sun L, Cheng C. Expression of CRM1 in human gliomas and its significance in p27 expression and clinical prognosis. Neurosurgery 2009; 65: 153-159, discussion $159-160$.

25 Walker CJ, Oaks JJ, Santhanam R, Neviani P, Harb JG, Ferenchak G et al. Preclinical and clinical efficacy of XPO1/CRM1 inhibition by the karyopherin inhibitor KPT-330 in Ph+ leukemias. Blood 2013; 122: 3034-3044.

26 Yao $Y$, Dong $Y$, Lin F, Zhao $H$, Shen Z, Chen P et al. The expression of CRM1 is associated with prognosis in human osteosarcoma. Oncol Rep 2009; 21: 229-235.

27 Kalid O, Toledo Warshaviak D, Shechter S, Sherman W, Shacham S. Consensus Induced Fit Docking (cIFD): methodology, validation, and application to the discovery of novel Crm1 inhibitors. J Comput Aided Mol Des 2012; 26 1217-1228.

28 Savona M, Garzon R, de Nully Brown P, Yee K, Lancet JE, Gutierrez M et al. Phase I Trial of Selinexor (KPT-330), a first-in-class oral Selective Inhibitor of Nuclear Export (SINE) in patients (pts) with advanced acute myelogenous leukemia (AML). 58th ASH Annual Meeting \& Exposition, New Orleans, LA, USA, December 7-10, 2013.

29 Rubnitz J, Kaufman R, Ribeiro RC, Klebanov B, Ellis J, Landesman Y et al. Phase study of Selinexor, a selective inhibitor of nuclear export, in combination with Fludarabine and Cytarabine in pediatric patients with relapsed or refractory AML. In: ASH Annual Meeting, Orlando, FL, USA, December 2015.

30 Fiedler W, Chromik J, Kebenko M, Thol F, Trummer A, Schünemann C et al. Selinexor, ARA-C and Idarubicin: an effective and tolerable combination in patients with relapsed/refractory AML: a multicenter phase II study. In: ASH Annual Meeting, Orlando, FL, USA, December 2015.

31 Chen C, Garzon R, Gutierrez M, Jacoby MA, Brown P, Flinn IW et al. Safety, efficacy, and determination of the recommended phase 2 dose for the oral Selective Inhibitor of Nuclear Export (SINE) Selinexor (KPT-330). In: ASH Annual Meeting, Orlando, FL, USA, December 2015.

32 Ranganathan P, Yu X, Na C, Santhanam R, Shacham S, Kauffman M et al. Preclinical activity of a novel CRM1 inhibitor in acute myeloid leukemia. Blood 2012; 120 1765-1773.

33 Schmidt J, Braggio E, Kortuem KM, Egan JB, Zhu YX, Xin CS et al. Genome-wide studies in multiple myeloma identify XPO1/CRM1 as a critical target validated using the selective nuclear export inhibitor KPT-276. Leukemia 2013; 27 2357-2365

34 Tai YT, Landesman Y, Acharya C, Calle Y, Zhong MY, Cea M et al. CRM1 inhibition induces tumor cell cytotoxicity and impairs osteoclastogenesis in multiple myeloma: molecular mechanisms and therapeutic implications. Leukemia 2014; 28: $155-165$.

35 Ranganathan P, Yu X, Santhanam R, Hofstetter J, Walker A, Walsh K et al. Decitabine priming enhances the antileukemic effects of exportin 1 (XPO1) selective inhibitor selinexor in acute myeloid leukemia. Blood 2015; 125: 2689-2692.

36 Zhong Y, El-Gamal D, Dubovsky JA, Beckwith KA, Harrington BK, Williams KE et al. Selinexor suppresses downstream effectors of B-cell activation, proliferation and migration in chronic lymphocytic leukemia cells. Leukemia 2014; 28: 1158-1163.

37 Hing ZA, Mantel R, Beckwith KA, Guinn D, Williams E, Smith LL et al. Selinexor is effective in acquired resistance to ibrutinib and synergizes with ibrutinib in chronic lymphocytic leukemia. Blood 2015; 125: 3128-3132.

38 Ishizawa J, Kojima K, McQueen T, Ruvolo V, Chachad D, Nogueras-Gonzalez $\mathrm{GM}$ et al. Mitochondrial profiling of acute myeloid leukemia in the 
assessment of response to apoptosis modulating drugs. PLoS One 2015; 10: e0138377.

39 Dick JE. Acute myeloid leukemia stem cells. Ann New York Acad Sci 2005; 1044: 1-5.

40 Etchin J, Montero J, Berezovskaya A, Le BT, Kentsis A, Christie AL et al. Activity of a selective inhibitor of nuclear export, selinexor (KPT-330), against AML-initiating cells engrafted into immunosuppressed NSG mice. Leukemia 2015; 30: 190-199.

41 Sarry JE, Murphy K, Perry R, Sanchez PV, Secreto A, Keefer C et al. Human acute myelogenous leukemia stem cells are rare and heterogeneous when assayed in NOD/SCID/IL2Rgammac-deficient mice. J Clin Invest 2011; 121: 384-395.

42 Eppert K, Takenaka K, Lechman ER, Waldron L, Nilsson B, van Galen P et al. Stem cell gene expression programs influence clinical outcome in human leukemia. Nat Med 2011; 17: 1086-1093.

43 Chen WC, Yuan JS, Xing Y, Mitchell A, Mbong N, Popescu AC et al. An integrated analysis of heterogeneous drug response in acute myeloid leukemia that enables the discovery of predictive biomarkers. Cancer Res 2016; 76: 1214-1224.

$44 \mathrm{Hu}$ Y, Smyth GK. ELDA: extreme limiting dilution analysis for comparing depleted and enriched populations in stem cell and other assays. J Immunol Methods 2009; 347: 70-78.
45 Patel JP, Gonen M, Figueroa ME, Fernandez H, Sun Z, Racevskis J et al. Prognostic relevance of integrated genetic profiling in acute myeloid leukemia. $N$ Engl J Med 2012; 366: 1079-1089.

46 Oguro H, Ding L, Morrison SJ. SLAM family markers resolve functionally distinct subpopulations of hematopoietic stem cells and multipotent progenitors. Cell Stem Cell 2013; 13: 102-116.

C) (1) This work is licensed under a Creative Commons AttributionBY NA SA Commercial-ShareAlike 4.0 International License. The images or other third party material in this article are included in the article's Creative Commons license, unless indicated otherwise in the credit line; if the material is not included under the Creative Commons license, users will need to obtain permission from the license holder to reproduce the material. To view a copy of this license, visit http:// creativecommons.org/licenses/by-nc-sa/4.0/

(c) The Author(s) 2017

Supplementary Information accompanies this paper on the Leukemia website (http://www.nature.com/leu) 